\title{
Influenza vaccination and myocarditis among patients receiving immune checkpoint inhibitors
}

Magid Awadalla ${ }^{*}$ DD, Doll Lauren Alexandra Golden', Syed S. Mahmood², Raza M. Alvi', Nathaniel D. Mercaldo', Malek Z. O. Hassan', Dahlia Banerji', Adam Rokicki', Connor Mulligan', Sean P. T. Murphy', Maeve Jones-O'Connor ${ }^{1}$, Justine V. Cohen ${ }^{3}$, Lucie M. Heinzerling ${ }^{4}$, Merna Armanious ${ }^{5}$, Ryan J. Sullivann, Rongras Damrongwatanasuk ${ }^{5}$, Carol L. Chen ${ }^{6}$, Dipti Gupta ${ }^{6}$, Michael C. Kirchberger ${ }^{4}$, Javid J. Moslehi ${ }^{7}$, Sachin P. Shah ${ }^{8}$, Sarju Ganatra ${ }^{8}$, Paaladinesh Thavendiranathan ${ }^{9}$, Muhammad A. Rizvi ${ }^{10}$, Gagan Sahni ${ }^{11}$, Alexander R. Lyon ${ }^{12}$, Carlo G. Tocchetti ${ }^{13}$, Valentina Mercurio ${ }^{13}$, Franck Thuny ${ }^{14}$, Stephane Ederhy ${ }^{15}$, Michael Mahmoudi ${ }^{16}$, Donald P. Lawrence ${ }^{3}$, John D. Groarke ${ }^{17}$, Anju Nohria ${ }^{17}$, Michael G. Fradley ${ }^{5}$, Kerry L. Reynolds ${ }^{3}$ and Tomas G. Neilan ${ }^{1,18}$

\section{Abstract}

Background: Influenza vaccination (FV) is recommended for patients with cancer. Recent data suggested that the administration of the FV was associated with an increase in immune-related adverse events (irAEs) among patients on immune checkpoint inhibitors (ICls). Myocarditis is an uncommon but serious complication of ICls and may also result from infection with influenza. There are no data testing the relationship between FV and the development of myocarditis on ICls.

Methods: Patients on ICls who developed myocarditis $(n=101)$ (cases) were compared to ICl-treated patients $(n=201)$ without myocarditis (controls). A patient was defined as having the FV if they were administered the FV from 6 months prior to start of $\mathrm{ICI}$ to anytime during $\mathrm{ICI}$ therapy. Alternate thresholds for FV status were also tested. The primary comparison of interest was the rate of FV between cases and controls. Patients with myocarditis were followed for major adverse cardiac events (MACE), defined as the composite of cardiogenic shock, cardiac arrest, hemodynamically significant complete heart block and cardiovascular death.

Results: The FV was administered to $25 \%$ of the myocarditis cases compared to $40 \%$ of the non-myocarditis ICltreated controls $(p=0.01)$. Similar findings of lower rates of FV administration were noted among myocarditis cases when alternate thresholds were tested. Among the myocarditis cases, those who were vaccinated had 3-fold lower troponin levels when compared to unvaccinated cases (FV vs. No FV: $0.12[0.02,0.47]$ vs. $0.40[0.11,1.26] \mathrm{ng} / \mathrm{ml}$, $p=0.02$ ). Within myocarditis cases, those administered the FV also had a lower rate of other irAEs when compared to unvaccinated cases ( 36 vs. $55 \% p=0.10$ ) including lower rates of pneumonitis ( 12 vs. $36 \%, p=0.03$ ). During follow-up (175 [IQR 89, 363] days), 47\% of myocarditis cases experienced a MACE. Myocarditis cases who received the FV were at a lower risk of cumulative MACE when compared to unvaccinated cases (24 vs. 59\%, $p=0.002$ ).

(Continued on next page)

\footnotetext{
*Correspondence: mawadalla@mgh.harvard.edu

${ }^{1}$ Cardiac MR PET CT Program, Department of Radiology, Massachusetts

General Hospital, 165 Cambridge Street, Suite 400, Boston, MA 02114, USA

Full list of author information is available at the end of the article
}

(c) The Author(s). 2019 Open Access This article is distributed under the terms of the Creative Commons Attribution 4.0 International License (http://creativecommons.org/licenses/by/4.0/), which permits unrestricted use, distribution, and

reproduction in any medium, provided you give appropriate credit to the original author(s) and the source, provide a link to the Creative Commons license, and indicate if changes were made. The Creative Commons Public Domain Dedication waiver (http://creativecommons.org/publicdomain/zero/1.0/) applies to the data made available in this article, unless otherwise stated. 
(Continued from previous page)

Conclusion: The rate of FV among ICl-related myocarditis cases was lower than controls on ICls who did not develop myocarditis. In those who developed myocarditis related to an $\mathrm{ICl}$, there was less myocardial injury and a lower risk of MACE among those who were administered the FV.

Keywords: Influenza vaccination, Immune checkpoint inhibitors, Myocarditis, Cancer, Immune-related adverse events, Major adverse cardiac events, Cardiovascular disease

\section{Introduction}

Immune checkpoint inhibitors (ICIs) have revolutionized the care of several groups of patients with advanced cancers [1]. These therapies are approved for use among patients with metastatic melanoma, non-small cell lung cancer, squamous carcinoma of the head and neck, renal cell carcinoma, Hodgkin's lymphoma, liver cancer, squamous cell of the skin and bladder cancer [2-8]. Checkpoint inhibitors are predominantly approved in late stage patients but, due to efficacy, are being expanded to adjuvant settings [9-13]. They work by releasing negative regulators of immune activation, thus facilitating the recognition of tumors by the immune system. As anticipated, activation of the immune system may result in immune-mediated adverse effects (irAEs) [14]. Myocarditis is an uncommon but serious immune complication of ICIs [15-22], myocarditis related to an ICI occurs early after initiation of ICIs [15] and the development of myocarditis after ICIs is associated with significant morbidity and mortality [15, 20-24]. The risk factors for the development of myocarditis among patients on ICI therapy are poorly understood $[16,25]$ and an improved understanding is needed. In this submission, we tested the effect of administration of the FV on the development of myocarditis. By way of background, there is controversy as to whether it is safe to administer the influenza vaccine to patients receiving immunotherapy and there are data in patients at risk of cardiovascular disease that the influenza vaccine may be protective. The national comprehensive cancer network (NCCN) guidelines recommend the FV in patients with hematologic or solid tumor malignancies, but there are no guidelines specific to those on immune therapy [26]. In a recent study, with 23 lung cancer patients on ICI (cases) and 11 agematched healthy controls, influenza vaccination was associated with a heightened immune and inflammatory response resulting in a high rate of general irAEs (52.2\%) [27]. In contrast, in a multi-center study with a broad range of cancers, FV administration was associated with a non-significant increase in overall survival among patients on ICl's [28] and in a single center retrospective study of over 500 patients, a similar rate of general irAEs were noted between vaccinated $(37.4 \%)$ and unvaccinated patients (42.6\%) [29]. However, the majority of the irAEs in that study $(87 \%)$ were ICI- related pneumonitis and there are no studies to date testing the association of the FV and development of ICI related myocarditis [29]. Therefore, the goal of this study is to test the association between FV and the development of myocarditis among patients on ICI's. This relationship between FV status and the development of myocarditis may be of additional importance as the development of influenza infection is also, albeit rarely, associated itself with an increased risk for myocarditis and major adverse cardiovascular events [30-32]. Additional goals included testing the effect of FV status on outcomes among those patients who develop myocarditis.

\section{Methods \\ Patients}

Cases were derived from a 16-center institutional registry, which was created to collate cases of ICI-related myocarditis. The cases were diagnosed between November 2013 and October 2018. Controls were derived from a single-center registry (Massachusetts General Hospital, Boston, Massachusetts) of all patients started on ICI in the same time interval who did not develop myocarditis. The number of patients treated with ICI therapy at Massachusetts General Hospital during the study period was confirmed by 2 independent researchers. Controls, in a 2:1 ratio, were randomly selected and not pre-selected to match cases on any variables. The study was approved by each center's institutional review board, and the requirement for written informed consent was waived.

\section{Covariates}

Data on covariates of interest were retrospectively extracted from electronic medical records and included standard demographics, cardiovascular risk factors, medication, and echocardiographic variables. Cancer-specific covariates included the cancer type, ICI treatment, prior cardiotoxic chemotherapy, and prior radiation therapy. Myocarditis specific covariates included clinical presentation, physical examination, cardiac biomarkers, and echocardiographic parameters.

\section{Definitions and outcome of interest}

The diagnosis of myocarditis was made by one of two standard methods; 1 . The presence of standard histological features present on endomyocardial biopsy or 
autopsy or 2. A guideline-recommended standardized scoring system which incorporates clinical, biomarker and cardiac imaging features [33]. Subjects were defined as having received the FV if they received the FV anytime from 6 months prior to starting ICI to receiving the FV while on ICI therapy. This time frame was chosen as numerous studies have shown the period of effectiveness of the vaccine ranges within different cohorts, but peaks at 4-6 months, after which significantly declines [34, 35]. The administration of FV was at the discretion of clinician involved in care and not performed as part of a study. Two alternate thresholds to define FV status were also tested. In a second analysis, we defined FV status based on receiving the FV anytime from 3 months prior to starting ICI to receiving the FV while on ICI therapy. In the third definition of FV status, the FV group was restricted to those who were administered the FV after starting on an ICI. The first comparison was between cases who developed myocarditis and controls who did not develop myocarditis, separated by FV status. Additional analyses performed were restricted only to myocarditis cases. Within the cases who developed myocarditis, we next tested the association between FV status and adverse cardiovascular outcomes after the development of myocarditis. Major adverse cardiac events (MACE) was defined, as per prior studies among patients on ICI, as a composite of cardiovascular death, cardiac arrest, cardiogenic shock, and hemodynamically significant complete heart block (CHB) [15]. In cases where cardiac arrest, cardiogenic shock, or CHB led to death, that case was counted as a cardiac death. Standard definitions were used for cardiovascular death [36], cardiac arrest [37], and cardiogenic shock [38]. Hemodynamically significant $\mathrm{CHB}$ was defined as a complete absence of atrial-to-ventricular conduction requiring a temporary pacemaker [39].

\section{Statistical analysis}

Continuous variables were summarized as either the mean \pm standard deviation (SD) or as the median and interquartile range (IQR), as appropriate, and categorical variables were presented as percentages. Comparisons by case status (case vs. control) and by flu vaccination status were compared using the Student's $t$-test for continuous variables or either the chi-square or Fisher's exact test for categorical variables. Kaplan Meier curves and the log-rank test were generated to quantify the relationship between FV and MACE-free survival. All statistical tests were 2 -sided and $5 \%$ was set as the level of significance. Statistical analysis was performed using R Version 3.5.1 (R foundation for statistical computing, Vienna, Austria).

\section{Results}

\section{Patient characteristics}

The mean age of patients $(n=101)$ who developed ICI-associated myocarditis was $67 \pm 18$ years with $72 \%$ being male (Table 1). The median time to onset of myocarditis from first ICI was 57 days (interquartile range 27-122 days). In comparison with controls $(n=201)$, myocarditis cases had a higher body mass index (Table 1); otherwise, there were no major differences in non-cancer variables between cases and controls. The most common presentations were chest pain and shortness of breath (Table 3). An echocardiogram was performed in 98\% (99/ 101) of cases; $41 \%$ (41/99) had a reduced ejection fraction (EF) $(<50 \%)$ and $59 \%$ had a preserved EF.

\section{Cancer and treatment characteristics}

The most common indications for ICI were melanoma and non-small cell lung cancer (Table 1). Compared to controls, the myocarditis cases were less likely to have had prior radiation therapy, taxol or carboplatin chemotherapy (Table 1). When compared to the control group without myocarditis, the myocarditis cases were also more likely to have received combination ICI therapy (Table 2). However, overall, most cases of myocarditis were being treated with concurrent single ICI therapy (72\%). A complete description of the ICI therapies between cases and controls separated by those on combination therapy or single therapy at presentation is shown in Table 2. The median follow-up time was 290 [IQR 139,543] days for controls, and 175 [89,363] days for myocarditis cases (Table 2). 50\% of the myocarditis cases had not experienced another ICI-related side effect. There was generally no difference in the overall prevalence of other ICI-related side effects between cases and controls; however, myocarditis cases who did have an additional previous immune-related side effect had higher rates of pneumonitis and neurological side effects (Table 2).

\section{Influenza vaccination}

Within 6 months prior to starting or during ICI treatment, $25 \%$ (25/101) of the myocarditis cases received the FV (median of 88 days, interquartile range 25-120 days). In comparison, $\mathrm{FV}$ was administered to $40 \%$ (80/201, $p=0.01$ for rate comparison) of controls on an ICI who did not develop myocarditis (median of 79 days, interquartile range of 43-170, Table 1). We also restricted the comparison of $\mathrm{FV}$ rates to cases from the institution where the controls were also derived (MGH). We found that in an analysis restricted to myocarditis cases at $\mathrm{MGH}$, the rate of FV among cases was $17 \%(5 / 30, p=0.02)$. Additional time-cut offs in the larger cohort were also tested to define whether a patient received the FV. In a second cut-off, we defined FV as having been administered the FV within 3 months prior to starting ICI treatment or during ICI therapy. When implementing this second time-cut off, $17 \%$ $(17 / 101)$ of the myocarditis cases $(31[6,85]$ days prior to ICI start) received the FV compared to $34 \%(69 / 201$, 
Table 1 Description of cases and controls

\begin{tabular}{|c|c|c|c|}
\hline & $\begin{array}{l}\text { Myocarditis } \\
(n=101)\end{array}$ & $\begin{array}{l}\text { Controls } \\
(n=201) \\
\end{array}$ & $P$ Value \\
\hline Age at start of $\mathrm{ICl}$, yrs & $67 \pm 18$ & $64 \pm 14$ & 0.15 \\
\hline Male & $73(72)$ & $129(64)$ & 0.16 \\
\hline \multicolumn{4}{|l|}{ CV risk factors } \\
\hline Current or prior smoking & $40(47)$ & $110(58)$ & 0.10 \\
\hline Hypertension & $59(60)$ & $115(61)$ & 0.88 \\
\hline Diabetes mellitus & $22(23)$ & $29(15)$ & 0.09 \\
\hline No CV risk factors & $23(23)$ & $40(20)$ & 0.56 \\
\hline Coronary artery disease & $12(13)$ & $24(13)$ & 0.86 \\
\hline Stroke & $7(8)$ & $22(12)$ & 0.32 \\
\hline Heart failure & $5(6)$ & $13(7)$ & 0.69 \\
\hline COPD & $12(14)$ & $25(13)$ & 0.87 \\
\hline Obstructive sleep apnea & $6(7)$ & $11(6)$ & 0.70 \\
\hline Chronic kidney disease $^{a}$ & $9(11)$ & $31(16)$ & 0.22 \\
\hline Body mass index, $\mathrm{kg} / \mathrm{m}^{2}$ & $28 \pm 7$ & $26 \pm 6$ & 0.01 \\
\hline \multicolumn{4}{|l|}{ Primary cancer type } \\
\hline Head and neck & $5(5)$ & $14(7)$ & 0.50 \\
\hline Hodgkin's lymphoma & $2(2)$ & $2(1)$ & 0.60 \\
\hline Melanoma & $44(44)$ & $100(50)$ & 0.31 \\
\hline Lung cancer & $17(17)$ & $35(17)$ & 1.00 \\
\hline Pancreatic & $2(2)$ & 0 & 0.11 \\
\hline Renal cell carcinoma & $6(6)$ & $3(1)$ & 0.07 \\
\hline Glioblastoma & $2(2)$ & $2(1)$ & 0.60 \\
\hline Other & $23(23)$ & $20(10)$ & 0.005 \\
\hline \multicolumn{4}{|l|}{ Prior chemotherapy or radiation } \\
\hline Radiation & $29(29)$ & $108(54)$ & $<0.001$ \\
\hline Anthracyclines & $6(6)$ & $3(1)$ & 0.07 \\
\hline Cyclophosphamide & $2(2)$ & $2(1)$ & 0.60 \\
\hline Gemcitabine & $5(5)$ & $8(4)$ & 0.77 \\
\hline Taxanes & $6(6)$ & $32(16)$ & 0.01 \\
\hline Carboplatin & $8(8)$ & $60(30)$ & $<0.001$ \\
\hline VEGF Inhibitors & $1(1)$ & $7(3)$ & 0.28 \\
\hline \multicolumn{4}{|l|}{ Pre-ICI home CV medications } \\
\hline Statin & $32(37)$ & $45(24)$ & 0.02 \\
\hline Aspirin & $23(26)$ & $42(22)$ & 0.43 \\
\hline Beta-blockers & $24(28)$ & $55(29)$ & 0.84 \\
\hline ACE inhibitors or ARB & $26(30)$ & $38(20)$ & 0.07 \\
\hline Calcium-channel blocker & $8(9)$ & $33(17)$ & 0.08 \\
\hline
\end{tabular}

Rate of influenza vaccination

6 months:

$\begin{array}{llll}6 \text { months prior to } \mathrm{ICl} \text { or on } \mathrm{ICl} & 25(25) & 80(40) & 0.01 \\ \text { Time of vaccination prior to } & 88[25,120] & 79[43,170] & 0.53 \\ \mathrm{ICl} \text {, days } & & & \end{array}$

Table 1 Description of cases and controls (Continued)

\begin{tabular}{|c|c|c|c|}
\hline & $\begin{array}{l}\text { Myocarditis } \\
(n=101)\end{array}$ & $\begin{array}{l}\text { Controls } \\
(n=201)\end{array}$ & $P$ Value \\
\hline \multicolumn{4}{|l|}{3 months: } \\
\hline 3 months prior to $\mathrm{ICl}$ or on $\mathrm{ICl}$ & $17(17)$ & $69(34)$ & 0.002 \\
\hline $\begin{array}{l}\text { Time of vaccination prior to } \\
\mathrm{ICl} \text {, days }\end{array}$ & $31[6,85]$ & $44[13,58]$ & 0.88 \\
\hline \multicolumn{4}{|l|}{ On ICI therapy only: } \\
\hline On $\mathrm{ICl}$ & $8(8)$ & $34(17)$ & 0.04 \\
\hline
\end{tabular}

$p=0.002$ for rate comparison) of controls $(44[13,58]$ days prior to ICI start, Table 1). A complete description comparing the myocarditis cases using the 3-month time-cut off stratified by FV status is presented in Additional file 1: Table S1. We additionally used a third cut-off time to define FV status. In this third cut-off, we defined FV as only those who were administered the FV while on ICI. When FV status was restricted to those administered the FV while on $\mathrm{ICI}$, the rates of $\mathrm{FV}$ in myocarditis cases during the period while on ICI therapy was $8 \%(8 / 101)$ compared to $17 \%(34 / 201)$ of controls who did not develop myocarditis $(p=0.04$, a complete description of comparisons using this final threshold is not shown). We also tested whether there was temporal pattern in myocarditis presentation. There was no difference found in the temporal pattern of presentation with myocarditis, with $31 \%$ occurring in Spring, 22\% in Summer, 21\% in Autumn and $26 \%$ in Winter $(p=0.31)$.

\section{Comparison within myocarditis cases of those that were and were not administered the FV}

When myocarditis cases who received the FV in the 6 months prior to ICI were compared to myocarditis cases who did not receive the FV, there was no difference with respect to age ( $69 \pm 8$ vs. $66 \pm 20$ years, $p=0.60)$, sex (male, 68 vs. $74 \%, p=0.58$ ), or cardiovascular risk factors (smoking history 48 vs. $47 \%, p=0.95$; hypertension 58 vs. $60 \%, p=0.42$; diabetes mellitus 30 vs. $21 \%, p=0.36$, Table 3). There was also no difference in the use of monotherapy or combined ICI treatment, as well as overall ICIs used among myocarditis cases when stratified by vaccination status. A complete description of the comparisons of ICI therapies between myocarditis cases who were and were not administered the FV is presented in Table 3. The occurrence of other irAEs was compared within the myocarditis cases, and $36 \%$ of cases vaccinated compared to $55 \%$ of unvaccinated cases had further immune side effects during treatment $(p=0.10)$. Cases administered the vaccination were not at 
Table 2 Baseline cancer demographics

\begin{tabular}{|c|c|c|c|}
\hline & $\begin{array}{l}\text { Cases } \\
(n=101) \\
\end{array}$ & $\begin{array}{l}\text { Controls } \\
(n=201) \\
\end{array}$ & $P$ value \\
\hline \multicolumn{4}{|l|}{ Single agent vs. combined } \\
\hline Combination & $28(28)$ & $14(7)$ & $<0.001$ \\
\hline Monotherapy & $73(72)$ & $177(93)$ & $<0.001$ \\
\hline \multicolumn{4}{|l|}{ Combined $\mathrm{ICl}$} \\
\hline $\begin{array}{l}\text { Ipilimumab (anti-CTLA4) + } \\
\text { nivolumab (anti-PD1) }\end{array}$ & $24(24)$ & $13(6)$ & $<0.001$ \\
\hline $\begin{array}{l}\text { Ipilimumab (anti-CTLA4) + } \\
\text { pembrolizumab (anti-PD1) }\end{array}$ & $1(1)$ & 0 & 0.33 \\
\hline $\begin{array}{l}\text { Tremelimumab (anti-CTLA4) + } \\
\text { avelumab (anti-PD1) }\end{array}$ & $1(1)$ & 0 & 0.33 \\
\hline $\begin{array}{l}\text { Tremelimumab (anti-CTLA4) + } \\
\text { durvalumab (anti-PD1) }\end{array}$ & $2(2)$ & $1(0)$ & 0.26 \\
\hline \multicolumn{4}{|l|}{ Monotherapy $\mid \mathrm{ICl}^{\mathrm{a}}$} \\
\hline Pembrolizumab (anti-PD1) & $35(35)$ & $62(31)$ & 0.50 \\
\hline Nivolumab (anti-PD1) & $25(25)$ & $85(42)$ & 0.003 \\
\hline Ipilimumab (anti-CTLA4) & $6(6)$ & $28(14)$ & 0.04 \\
\hline Tremelimumab (anti-CTLA4) & $1(1)$ & 0 & 0.33 \\
\hline Atezolizumab (anti-PDL1) & $6(6)$ & $2(1)$ & 0.02 \\
\hline Avelumab (anti-PDL1) & 0 & 0 & 1.00 \\
\hline Durvalumab (anti-PDL1) & 0 & 0 & 1.00 \\
\hline \multicolumn{4}{|l|}{ Overall types of $\mathrm{ICl}$} \\
\hline Any anti-PD1 & $85(84)$ & $160(80)$ & 0.34 \\
\hline Any anti-CTLA4 & $35(35)$ & $42(21)$ & 0.01 \\
\hline Any anti-PDL1 & $9(9)$ & $3(1)$ & 0.003 \\
\hline Days of follow-up [IQR] & $175[89,363]$ & $290[139,543]$ & $<0.001$ \\
\hline \multicolumn{4}{|c|}{ Other immune side effects during treatment ${ }^{b}$} \\
\hline No other immune side effects & $51(50)$ & $86(43)$ & 0.20 \\
\hline Hypophysitis/pituitary/adrenal & $6(6)$ & $14(7)$ & 0.74 \\
\hline Pneumonitis & $30(30)$ & $24(12)$ & $<0.001$ \\
\hline Hepatitis & $8(8)$ & $11(5)$ & 0.41 \\
\hline Colitis & $9(9)$ & $27(13)$ & 0.25 \\
\hline Dermatitis & $6(6)$ & $5(2)$ & 0.19 \\
\hline Neurological & $11(11)$ & $4(2)$ & 0.001 \\
\hline Gastritis & $3(3)$ & $5(2)$ & 1.00 \\
\hline
\end{tabular}

Values are $\mathrm{n}(\%)$ or mean $\pm \mathrm{SD}$. All cases with ICl-associated myocarditis had ICl permanently discontinued. alf most recent ICl therapy was monotherapy. ${ }^{\mathrm{b}}$ More than one immune side effect may occur. Anti-CTLA4 anti-cytotoxic T-lymphocyte-associated protein 4, anti-PD1 anti-programmed cell death protein 1, anti-PDL1 anti-programmed death-ligand 1, ICl immune checkpoint inhibitors

increased risk of other immune side effects during treatment (FV vs. no FV, hypophysitis 4 vs. $7 \%, p=1.00$; hepatitis 4 vs. $9 \%, p=0.68$; colitis 8 vs. $9 \%, p=1.00$; dermatitis 0 vs. $8 \%, p=0.33$; neurological 4 vs. $13 \%, p=$ 0.28 or gastritis 0 vs. $4 \%, p=0.57$ (Table 3 )). In contrast, myocarditis cases administered the FV were less likely to have prior ICI-related pneumonitis ( 12 vs. $36 \%, p=0.03$ ) (Table 3 ). When stratifying the groups by FV status, there was no difference in the LVEF $(46 \pm 15$ vs. $50 \pm$ $16 \%, p=0.28$, Table 3) but serum troponin, a measure of myocardial injury, was higher among cases who did not receive the FV. Specifically, when compared to unvaccinated cases, cases administered the FV had a 3-fold lower troponin $\mathrm{T}$ level $(0.12$ [0.02, 0.47] vs. 0.40 [0.11, $1.26] \mathrm{ng} / \mathrm{ml}, p=0.02$ ) (Table 3).

\section{Major adverse cardiac events}

The median follow-up of myocarditis cases was 175 days (interquartile range 89 to 363 days] (Table 2) and during this follow-up period, $47 \%$ (47/101) of all myocarditis cases experienced a MACE: CHB $(n=16)$, cardiogenic shock $(n=17)$, cardiac arrest $(n=15)$, or cardiovascular death $(n=32$, Table 3$)$. Myocarditis cases who received the FV were at a lower risk of cumulative MACE when compared to unvaccinated cases (cumulative MACE 24 vs. $59 \%, p=0.002$ ) (Fig. 1). When the individual components of MACE were compared, vaccinated cases were less likely to have a cardiovascular death when compared to cases not administered the flu vaccine ( 36 vs. $72 \%, p=0.04$, Table 3). The rates of the other individual components were non-significantly lower among those administered the FV: complete heart block ( 9 vs. $19 \%$, FV vs. no FV, $p=0.35$ ), cardiogenic shock ( 9 vs. $20 \%, p=0.35$ ), or cardiac arrest ( 9 vs. $17 \%, p=0.51$, Table 3 ).

\section{Discussion}

We tested the association between FV and the development of myocarditis among patients on ICIs, and the effect of FV status on presentation and outcomes among patients who develop ICI-myocarditis. In our retrospective study of 101 ICI-related myocarditis cases, we found that $25 \%$ were vaccinated against influenza. In comparison, rate of vaccination for influenza was higher (40\%) among 201 control patients on ICIs who did not develop myocarditis. This first analysis included those administered the FV from 6 months prior to commencing ICI therapy or during therapy. Similar findings of lower rates of vaccination against flu among myocarditis cases were noted when using a 3-month cut-off or, when restricting FV to administration during ICI therapy only and restricting to cases from the same institution from where the controls were derived. Among myocarditis cases, serum troponin, a measure of myocardial injury used to detect myocarditis, was 3 -fold higher among myocarditis cases not administered the FV. This increase in serum troponin among unvaccinated myocarditis cases was associated with an increase in subsequent major adverse cardiac events. Specifically, during follow up, the rate of cumulative MACE among unvaccinated cases was more than double the adverse event rate seen among myocarditis cases administered the FV. Additional parallel findings of importance related to pneumonitis were noted 
Table 3 Comparison of Myocarditis cases with and without Flu vaccination (FV)

\begin{tabular}{|c|c|c|c|}
\hline & $\begin{array}{l}\mathrm{FV} \\
(n=25)\end{array}$ & $\begin{array}{l}\text { No FV } \\
(n=76)\end{array}$ & P Value \\
\hline Age at start of $\mathrm{ICl}$, yrs & $69 \pm 8$ & $66 \pm 20$ & 0.60 \\
\hline Male & $17(68)$ & $56(74)$ & 0.58 \\
\hline \multicolumn{4}{|l|}{ CV risk factors } \\
\hline Current or prior smoking & $10(48)$ & $30(47)$ & 0.95 \\
\hline Hypertension & $14(58)$ & $45(60)$ & 0.89 \\
\hline Diabetes mellitus & $7(30)$ & $15(21)$ & 0.36 \\
\hline No CV risk factors & $4(16)$ & $19(25)$ & 0.35 \\
\hline Coronary artery disease & $3(15)$ & $9(13)$ & 0.73 \\
\hline Stroke & $1(5)$ & $6(8)$ & 1.00 \\
\hline Heart failure & $1(5)$ & $4(6)$ & 1.00 \\
\hline COPD & $5(28)$ & $7(10)$ & 0.12 \\
\hline Obstructive sleep apnea & 0 & $6(9)$ & 0.60 \\
\hline Chronic kidney disease $^{a}$ & $2(11)$ & $7(10)$ & 1.00 \\
\hline Body mass index, $\mathrm{kg} / \mathrm{m}^{2}$ & $28 \pm 5$ & $28 \pm 7$ & 0.90 \\
\hline \multicolumn{4}{|l|}{ Primary cancer type } \\
\hline Head and neck & 0 & $5(7)$ & 0.33 \\
\hline Hodgkin's lymphoma & 0 & $2(3)$ & 1.00 \\
\hline Melanoma & $12(48)$ & $32(42)$ & 0.61 \\
\hline Lung cancer & $6(24)$ & $11(14)$ & 0.35 \\
\hline Pancreatic & $2(8)$ & 0 & 0.06 \\
\hline Renal cell carcinoma & $2(8)$ & $4(5)$ & 0.64 \\
\hline Glioblastoma & 0 & $2(3)$ & 1.00 \\
\hline Other & $3(12)$ & $20(26)$ & 0.18 \\
\hline \multicolumn{4}{|l|}{ Prior chemotherapy or radiation } \\
\hline Radiation & $4(16)$ & $25(33)$ & 0.11 \\
\hline Anthracyclines & $1(4)$ & $5(7)$ & 1.00 \\
\hline Cyclophosphamide & $1(4)$ & $1(1)$ & 0.44 \\
\hline Gemcitabine & $2(8)$ & $3(4)$ & 0.60 \\
\hline Taxanes & $2(8)$ & $4(5)$ & 0.64 \\
\hline Carboplatin & $2(8)$ & $6(8)$ & 1.00 \\
\hline VEGF Inhibitors & 0 & $1(1)$ & 1.00 \\
\hline \multicolumn{4}{|l|}{ Single agent vs. combined $\mathrm{ICI}$} \\
\hline Combination & $8(32)$ & $20(26)$ & 0.61 \\
\hline Monotherapy & $17(68)$ & $56(74)$ & 0.61 \\
\hline \multicolumn{4}{|l|}{ Combined ICI } \\
\hline Ipilimumab + nivolumab & $8(32)$ & $16(21)$ & 0.27 \\
\hline Ipilimumab + pembrolizumab & 0 & $1(1)$ & 1.00 \\
\hline Tremelimumab + avelumab & 0 & $1(1)$ & 1.00 \\
\hline Tremelimumab + durvalumab & 0 & $2(3)$ & 1.00 \\
\hline \multicolumn{4}{|l|}{ Monotherapy $|C|^{b}$} \\
\hline Pembrolizumab (anti-PD1) & $7(28)$ & $28(37)$ & 0.42 \\
\hline Nivolumab (anti-PD1) & $7(28)$ & $18(24)$ & 0.66 \\
\hline Ipilimumab (anti-CTLA4) & $2(8)$ & $4(5)$ & 0.64 \\
\hline
\end{tabular}

Table 3 Comparison of Myocarditis cases with and without Flu vaccination (FV) (Continued)

\begin{tabular}{llll}
\hline & $F V$ & No FV & P Value \\
& $(n=25)$ & $(n=76)$ & \\
\hline Tremelimumab (anti-CTLA4) & $1(4)$ & 0 & 0.25 \\
Atezolizumab (anti-PDL1) & 0 & $6(8)$ & 0.33 \\
Avelumab (anti-PDL1) & 0 & 0 & 1.00 \\
Durvalumab (anti-PDL1) & 0 & 0 & 1.00 \\
Overall types of ICl & & & \\
Any anti-PD1 & $22(88)$ & $63(83)$ & 0.75 \\
Any anti-CTLA4 & $11(44)$ & $24(32)$ & 0.33 \\
Any anti-PDL1 & 0 & $9(12)$ & 0.11 \\
Days of follow-up [IQR] & 223 [111, 324] & 162 [86, 364] & 0.32 \\
Other immune side effects during treatment & & \\
No other immune side effects & $16(64)$ & $34(45)$ & 0.10 \\
Hypophysitis/pituitary/adrenal & $1(4)$ & $5(7)$ & 1.00 \\
Pneumonitis & $3(12)$ & $26(36)$ & 0.03 \\
Hepatitis & $1(4)$ & $7(9)$ & 0.68 \\
Colitis & $2(8)$ & $7(9)$ & 1.00 \\
Dermatitis & 0 & $6(8)$ & 0.33 \\
Neurological & $1(4)$ & $10(13)$ & 0.28 \\
Gastritis & $0(0)$ & $3(4)$ & 0.57
\end{tabular}

Myocarditis presentation ${ }^{c}$

$\begin{array}{llll}\text { Chest pain } & 15(60) & 50(66) & 0.60 \\ \text { Shortness of breath } & 6(25) & 21(28) & 0.75 \\ \text { Orthopnea } & 6(26) & 18(24) & 0.86 \\ \text { Paroxysmal nocturnal dyspnea } & 2(9) & 7(9) & 1.00 \\ \text { Fatigue } & 8(40) & 27(46) & 0.65\end{array}$

Admission examination

$\begin{array}{llll}\text { Jugular venous distension } \quad 8(32) & 24(32) & 1.00\end{array}$

Crackles on lung exam $\quad 8(32) \quad 36(47) \quad 0.25$

Admission vitals

Heart rate, beats/min $\quad 92 \pm 16 \quad 89 \pm 24 \quad 0.67$

Systolic blood pressure, $\quad 126 \pm 17 \quad 126 \pm 21 \quad 0.89$

$\mathrm{mmHg}$

Diastolic blood pressure, $\quad 70 \pm 10 \quad 72 \pm 11 \quad 0.48$ $\mathrm{mmHg}$

Respiratory rate, rate, min $\quad 19 \pm 2 \quad 0.38$

Oxygen requirement and delivery ${ }^{\mathrm{d}}$

$\begin{array}{llll}\text { Room air } & 13(72) & 51(75) & 0.61 \\ \text { Nasal cannula } & 5(28) & 13(19) & 0.61 \\ \text { lntubated } & 0 & 4(6) & 0.61\end{array}$

Echocardiography, myocarditis admission

$\begin{array}{llll}\text { LVEF }^{\mathrm{e}}, \% & 46 \pm 15 & 50 \pm 16 & 0.28 \\ \text { LVIDD, mm } & 45 \pm 11 & 48 \pm 6 & 0.15\end{array}$

Admission cardiac enzymes

Troponin T, ng/ml

$0.12[0.02,0.47] \quad 0.40[0.11,1.26] \quad 0.02$ 
Table 3 Comparison of Myocarditis cases with and without Flu vaccination (FV) (Continued)

\begin{tabular}{|c|c|c|c|}
\hline & $\begin{array}{l}\text { FV } \\
(n=25)\end{array}$ & $\begin{array}{l}\text { No FV } \\
(n=76)\end{array}$ & $P$ Value \\
\hline BNP or NT-pro BNP, pg/ml & $568[421,987]$ & $600[215,4275]$ & 0.82 \\
\hline \multicolumn{4}{|l|}{ Outcomes: $M A C E^{f}$} \\
\hline Cumulative MACE & $6(24)$ & $45(59)$ & 0.002 \\
\hline Complete heart block & $2(9)$ & $14(19)$ & 0.35 \\
\hline Cardiogenic shock & $2(9)$ & $15(20)$ & 0.35 \\
\hline Cardiac arrest & $2(9)$ & $13(17)$ & 0.51 \\
\hline Cardiovascular death & $4(36)$ & $28(72)$ & 0.04 \\
\hline
\end{tabular}

Values are mean $\pm \mathrm{SD}$ or $\mathrm{n}(\%)$, or median [interquartile range]. ${ }^{\mathrm{a}} \mathrm{Chronic}$ kidney disease $=$ glomerular filtration rate $<60 \mathrm{ml} / \mathrm{min} / 1.73 \mathrm{~m}^{2}$. blf most recent $\mathrm{ICl}$ therapy was monotherapy. ${ }^{\mathrm{C}} \mathrm{Can}$ include more than $1 .{ }^{\mathrm{d}} \mathrm{Of}$ available cases (18 vaccinated, 69 unvaccinated). ${ }^{\mathrm{e}}$ All vaccinated cases [25] and 74 of the 76 unvaccinated cases had an admission echocardiogram. ${ }^{\mathrm{f}}$ Cases may have had more than one MACE, but only first event encountered was included in analysis. $C V$ cardiovascular, ICl immune checkpoint inhibitors, anti-CTLA4 anti-cytotoxic T-lymphocyte-associated protein 4, anti-PD1 anti-programmed cell death protein 1, anti-PDL1 anti-programmed death-ligand 1, LVEF left ventricular ejection fraction, $L V I D D$ left ventricular internal dimension diameter, $B N P$ brain natriuretic peptide, NT- pro BNP N-terminal pro BNP

that were not the primary focus of this paper. As compared to controls who did not develop myocarditis, the rates of pneumonitis were higher among myocarditis cases; and in analyses restricted to myocarditis cases, the rates of pneumonitis were higher among those cases not administered the FV.

Data testing the association between FV status and immune-mediated adverse events among patients on ICI therapy have provided conflicting results. In a single center study among patients with lung cancer, influenza vaccination during treatment with anti-PD1 induced an adequate serological protection from influenza, an increased inflammatory response and heightened the risk for immune-related adverse events [27]. There are differences in our study and that prior study that may explain the discordant findings. Specifically, we included patients

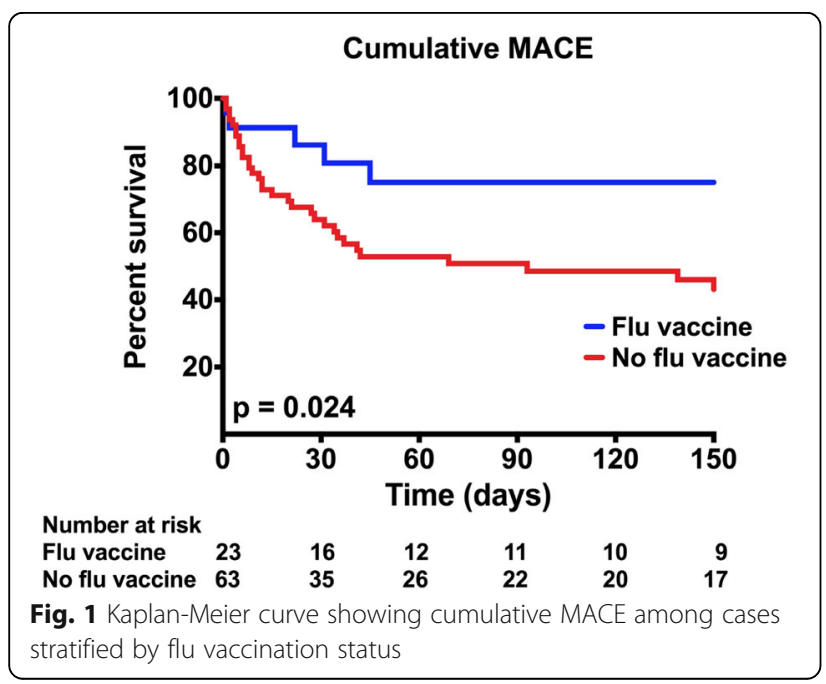

with all types of cancers who were on an ICI, we included all types of ICI therapies and we only primarily focused on one type of adverse event, myocarditis. We focused on myocarditis for the following four reasons: 1) myocarditis is an uncommon but serious complication of ICI therapy, 2) myocarditis can occur among patients with active influenza infection, 3) vaccination against influenza has been associated with a reduction in cardiovascular events in broad populations $[40,41], 4)$ the risk for cardiovascular events is increased broadly among patients with cancer, Data among broad populations showing a beneficial effect of FV on cardiovascular events are robust. For example, in a large meta-analysis of randomized controlled trials of nearly 7000 patients, the administration of $\mathrm{FV}$ was associated with a lower risk of MACE [30]. Other studies have also suggested that the use of the FV vaccine may be safe among patients on an ICI. Specifically, a recent single center study suggested that the seasonal influenza vaccination is safe and may be beneficial for patients on ICI with reduced rates of hospital admissions from flu-related and immunotherapy-related adverse events [29]. This latter study also included patients with all types of cancer and ICI therapies.

In supportive findings of a protective effect of $\mathrm{FV}$ among patients on an ICI, we found that biomarkers of risk in general myocarditis and for adverse events among patients who develop ICI myocarditis were higher among unvaccinated cases. We previously noted that serum troponin, a sensitive marker for myocardial injury is elevated among most patients with ICI myocarditis and the degree of elevation of serum troponin is a predictor of adverse cardiovascular events among patients who develop myocarditis on an ICI [15]. In this current study, troponin levels were higher among unvaccinated cases with ICI myocarditis compared to cases with myocarditis who were administered the FV. We also noted that serious adverse cardiac events were increased among patients who developed ICI myocarditis and were not previously administered the FV.

Although not the primary focus of this paper our findings regarding other immune-related adverse events, specifically pneumonitis, merit discussion. Our rates of any grade of other irAEs were 36 and $55 \%$ in the vaccinated and unvaccinated cases, respectively. These are comparable to the $37 \%$ irAEs in the vaccinated group and $43 \%$ in the unvaccinated group reported in the discussed single center study showing a protective effect of FV [29]. However, these rates are still lower than the rate of $52.2 \%$ of previously vaccinated patients developing any grade irAEs in the study of Läubli and colleagues [27]. We found higher rates of pneumonitis in the population without FV, Pneumonitis and pneumonia are important causes of influenza-associated morbidity and mortality among broad populations $[42,43]$ and FV has 
been shown to reduce morbidity and mortality among at-risk individuals $[40,43]$, including patients with cancer [44-46]. In our cohort, vaccinated cases had lower rates of immune-related pneumonitis compared to unvaccinated cases. This may be explained by the protective nature of the FV against pneumonitis and pneumonia and support the need for prospective randomized studies in this at-risk population.

This study has some limitations that merit discussion. This is the largest registry of patients with ICI-myocarditis; however, this was a retrospective case-control study where cases were derived from multiple institutions and controls were derived from a single institution. To address this, we also compared the rates of FV within cases and controls from the same institution and found similar results of a lower rate of FV among patients on an ICI who got myocarditis. Additionally, as this was a retrospective study, the type of influenza vaccination, the specific antibody titers and measures of inflammatory response were not recorded. Also, the choice of whether patients were administered the FV was at the discretion of the clinician involved in their care, which differed between the centers but also locally within each center. Ideally to test the association of the FV and ICI myocarditis, a prospective study comparing all ICI patients with and without FV who develop myocarditis, or a randomized clinical trial would be warranted. However, with a low incidence rate of ICI-myocarditis $(\sim 0.5-1.0 \%$ or less) $[14,15,20,21]$, to test this association adequately, a large cohort of subjects would be required. In addition, there are currently no available systematic screening approaches for myocarditis among patients on ICIs and diagnosis is based on physician's suspicion. Therefore, a prospective approach may also lead to an underestimation in the incidence of myocarditis and the effect of the FV. Finally, this study does not provide a mechanism by which the FV may be protective. Indeed, the mechanism underlying the protective effect of the FV against cardiovascular events in the general population is also unclear [30], but potential mechanisms include rupture of a vulnerable atherosclerotic plaque, heart failure, or, relevant to this study, myocarditis [32, 47-49]. Direct involvement of influenza in the myocardium, leading to myocarditis, is uncommon with rates of up to $10 \%$ reported depending on methods of detection used [31] and influenza infection can cause myocarditis by direct cytolysis of the myocyte causing necrosis, but also the host immune response to the virus may play an important role [50].

\section{Conclusion}

In summary, the administration of the FV was not associated with an increased risk of subsequent myocarditis among patients on ICI. In contrast, rates of influenza vaccination were lower among patients who did develop myocarditis on ICI, and the influenza vaccine was associated with a lower rate of ICI-related pneumonitis. At presentation, myocarditis cases administered the FV had lower troponin levels and, in follow-up, had lower rates of cumulative MACE. There is a clear need to establish the safety status of influenza vaccination among cancer patients treated with ICIs, as our data suggest that it may be protective. Further large studies are warranted to test and validate these important findings.

\section{Additional file}

Additional file 1: Table S1. (3-month cut-off): Comparison of Myocarditis cases with $^{\ddagger}$ and without Flu vaccination (FV). (DOCX $22 \mathrm{~kb}$ )

\section{Acknowledgments \\ Not applicable.}

\section{Funding}

Dr. S. S. Mahmood has been supported by the Sarnoff Cardiovascular Research Foundation. Dr. R.J. Sullivan was supported, in part, through the National Institutes of Health (NIH)/ National Cancer Institute (NCI) (RO1CA229851, UH2CA207355, R01CA193970). Dr. C.L. Chen, and Dr. D. Gupta were supported, in part, through the NIH/NCI P30CA008748. Dr. P. Thavendiranathan was supported, in part, through the Canadian Institutes of Health Research New Investigator Award (FRN 147814). Dr. C.G. Tocchetti was supported by a Ricerca di Ateneo/Federico II University grant. Dr. T.G. Neilan was supported, in part, through the Kohlberg Foundation, an American Heart Association Fellow to Faculty Award (12FTF12060588), NIH/NHLBI (1R01HL130539-01A1,

1R01HL137562-01A1, and K24HL113128-06), and NIH/Harvard Center for AIDS Research (P30 Al060354). The study was not funded.

\section{Availability of data and materials}

Not applicable.

\section{Authors' contributions}

MA drafted the manuscript with guidance from TGN. All authors participated in the conceptualization, writing, review, and revision of this manuscript. In addition, all authors have read and approved the final version of this manuscript.

Author's information

Not applicable.

Ethics approval and consent to participate

The Institutional Review Boards at each center granted a waiver of informed consent for this study.

\section{Consent for publication}

Not applicable.

\section{Competing interests}

Dr. Mahmood has received consultancy fees from OMR Globus, Alpha Detail, and Opinion Research Team. Dr. Nohria has received research support from Amgen; and has been a consultant for Takeda Oncology. Dr. Heinzerling has received consultancy, advisory board, and speaker fees from MSD, BMS, Roche, Novartis, Amgen, and Curevac. Dr. Sullivan has been a consultant to Merck and Novartis. Dr. Moslehi has served as a consultant/advisor for Novartis, Pfizer, Bristol-Myers Squibb, Takeda/Millennium, Ariad, Acceleron, Vertex, Incyte, Rgenix, Verastem, Pharmacyclics, StemCentRx, Heat Biologics, Daiichi-Sankyo, and Regeneron. Dr. Tocchetti has received speaker fees from Alere. Dr. Groarke has received research support from Amgen. Dr. Neilan has received advisory fees from Takeda and Parexel. All other authors have reported that they have no relationships relevant to the contents of this paper to disclose. 


\section{Publisher's Note}

Springer Nature remains neutral with regard to jurisdictional claims in published maps and institutional affiliations.

\begin{abstract}
Author details
'Cardiac MR PET CT Program, Department of Radiology, Massachusetts General Hospital, 165 Cambridge Street, Suite 400, Boston, MA 02114, USA. ${ }^{2}$ Cardiology Division, New York-Presbyterian Hospital, Weill Cornell Medical Center, New York, NY, USA. ${ }^{3}$ Division of Oncology and Hematology, Department of Medicine, Massachusetts General Hospital, Boston, MA, USA. ${ }^{4}$ Department of Dermatology, University Hospital Erlangen,

Friedrich-Alexander-University Erlangen-Nurnberg (FAU), Erlangen, Germany. ${ }^{5}$ Cardio-Oncology Program, H. Lee Moffitt Cancer Center \& Research Institute and University of South Florida Division of Cardiovascular Medicine, Tampa, FL, USA. ${ }^{6}$ Cardiology Division, Memorial Sloan Kettering Cancer Center, Weill Cornell Medical College, New York, NY, USA. ${ }^{7}$ Cardio-Oncology Program, Vanderbilt University Medical Center, Nashville, TN, USA. ${ }^{8}$ Cardiology Division, Lahey Hospital \& Medical Center, Burlington, MA, USA. ${ }^{9}$ Ted Rogers Program in Cardiotoxicity Prevention, Peter Munk Cardiac Center, Division of Cardiology Toronto General Hospital, University of Toronto, Toronto, Ontario, Canada. ${ }^{10}$ Division of Oncology and Hematology, Department of Medicine, Lehigh Valley Hospital, Allentown, PA, USA. "'The Mount Sinai Hospital, New York, NY, USA. ${ }^{12}$ Cardio-Oncology Program, Royal Brompton Hospital, London, UK. ${ }^{13}$ Department of Translational Medical Sciences, Federico II University, Naples, Italy. ${ }^{14}$ Cardiovascular Division, Department of Medicine, Aix-Marseille Universite, Marseille, France. ${ }^{15}$ Cardio-Oncology Program, Division of Cardiology, Hopitaux Universitaires est Paris, Paris, France. ${ }^{16}$ Division of Cardiology, Department of Medicine, Southampton General Hospital, Southampton, UK. ${ }^{17}$ Cardio-Oncology Program, Division of Cardiology, Department of Medicine, Brigham and Women's Hospital, Boston, MA, USA. ${ }^{18}$ Cardio-Oncology Program, Division of Cardiology, Department of Medicine, Massachusetts General Hospital, Boston, MA, USA.
\end{abstract}

Received: 12 December 2018 Accepted: 13 February 2019 Published online: 22 February 2019

\section{References}

1. Sharma P, Allison JP. Immune checkpoint targeting in Cancer therapy: towards combination strategies with curative potential. Cell. 2015;161(2): 205-14.

2. Borghaei H, Paz-Ares L, Horn L, Spigel DR, Steins M, Ready NE, et al. Nivolumab versus docetaxel in advanced nonsquamous non-small-cell lung Cancer. N Engl J Med. 2015;373(17):1627-39.

3. Ansell SM, Lesokhin AM, Borrello I, Halwani A, Scott EC, Gutierrez M, et al. PD-1 blockade with nivolumab in relapsed or refractory Hodgkin's lymphoma. N Engl J Med. 2015;372(4):311-9.

4. Hodi FS, O'Day SJ, McDermott DF, Weber RW, Sosman JA, Haanen JB, et al. Improved survival with ipilimumab in patients with metastatic melanoma. N Engl J Med. 2010;363(8):711-23.

5. Motzer RJ, Escudier B, McDermott DF, George S, Hammers HJ, Srinivas S, et al. Nivolumab versus Everolimus in advanced renal-cell carcinoma. N Engl J Med. 2015:373(19):1803-13.

6. Ferris RL, Blumenschein G, Fayette J, Guigay J, Colevas AD, Licitra L, et al. Nivolumab for Recurrent Squamous-Cell Carcinoma of the Head and Neck. N Engl J Med. 2016;375(19):1856-67.

7. Topalian SL, Hodi FS, Brahmer JR, Gettinger SN, Smith DC, McDermott DF, et al. Safety, activity, and immune correlates of anti-PD-1 antibody in cancer. N Engl J Med. 2012;366(26):2443-54.

8. Rosenberg JE, Hoffman-Censits J, Powles T, van der Heijden MS, Balar AV, Necchi A, et al. Atezolizumab in patients with locally advanced and metastatic urothelial carcinoma who have progressed following treatment with platinumbased chemotherapy: a single-arm, multicentre, phase 2 trial. Lancet Lond Engl. 2016;387(10031):1909-20.

9. Eggermont AMM, Blank CU, Mandala M, Long GV, Atkinson V, Dalle S, et al. Adjuvant Pembrolizumab versus placebo in resected stage III melanoma. N Engl J Med. 2018;378(19):1789-801.

10. Eggermont AMM, Chiarion-Sileni V, Grob J-J, Dummer R, Wolchok JD, Schmidt $\mathrm{H}$, et al. Adjuvant ipilimumab versus placebo after complete resection of high-risk stage III melanoma (EORTC 18071): a randomised, double-blind, phase 3 trial. Lancet Oncol. 2015;16(5):522-30.
11. Antonia SJ, Villegas A, Daniel D, Vicente D, Murakami S, Hui R, et al. Durvalumab after Chemoradiotherapy in Stage III Non-Small-Cell Lung Cancer. N Engl J Med. 2017;377(20):1919-29.

12. Atkins MB, Clark JI, Quinn DI. Immune checkpoint inhibitors in advanced renal cell carcinoma: experience to date and future directions. Ann Oncol Off J Eur Soc Med Oncol. 2017;28(7):1484-94.

13. Hanna N, Johnson D, Temin S, Baker S, Brahmer J, Ellis PM, et al. Systemic therapy for stage IV non-small-cell lung Cancer: American Society of Clinical Oncology clinical practice guideline update. J Clin Oncol Off J Am Soc Clin Oncol. 2017;35(30):3484-515.

14. Postow MA, Sidlow R, Hellmann MD. Immune-Related Adverse Events Associated with Immune Checkpoint Blockade. N Engl J Med. 2018; 378(2):158-68.

15. Mahmood SS, Fradley MG, Cohen JV, Nohria A, Reynolds KL, Heinzerling LM, et al. Myocarditis in patients treated with immune checkpoint inhibitors. J Am Coll Cardiol. 2018;71(16):1755-64.

16. Neilan TG, Rothenberg ML, Amiri-Kordestani L, Sullivan RJ, Steingart RM, Gregory W, et al. Myocarditis associated with immune checkpoint inhibitors: an expert consensus on data gaps and a call to action. Oncologist. 2018; 23(8):874-8.

17. Ganatra S, Neilan TG. Immune checkpoint inhibitor-associated myocarditis. Oncologist. 2018;23(8):879-86.

18. Moreira A, Loquai C, Pföhler C, Kähler KC, Knauss S, Heppt MV, et al. Myositis and neuromuscular side-effects induced by immune checkpoint inhibitors. Eur J Cancer Oxf Engl 1990. 2018;106:12-23.

19. Tajmir-Riahi A, Bergmann T, Schmid M, Agaimy A, Schuler G, Heinzerling L. Life-threatening Autoimmune Cardiomyopathy Reproducibly Induced in a Patient by Checkpoint Inhibitor Therapy. J Immunother Hagerstown Md 1997. 2018;41(1):35-8.

20. Varricchi G, Galdiero MR, Tocchetti CG. Cardiac toxicity of immune checkpoint inhibitors: cardio-oncology meets immunology. Circulation. 2017;136(21):1989-92.

21. Johnson DB, Balko JM, Compton ML, Chalkias S, Gorham J, Xu Y, et al. Fulminant myocarditis with combination immune checkpoint blockade. N Engl J Med. 2016;375(18):1749-55.

22. Moslehi JJ, Salem J-E, Sosman JA, Lebrun-Vignes B, Johnson DB. Increased reporting of fatal immune checkpoint inhibitor-associated myocarditis. Lancet Lond Engl. 2018;391(10124):933.

23. Ammirati E, Cipriani M, Lilliu M, Sormani P, Varrenti M, Raineri C, et al. Survival and left ventricular function changes in fulminant versus nonfulminant acute myocarditis. Circulation. 2017;136(6):529-45.

24. Salem J-E, Manouchehri A, Moey M, Lebrun-Vignes B, Bastarache L, Pariente A, et al. Cardiovascular toxicities associated with immune checkpoint inhibitors: an observational, retrospective, pharmacovigilance study. Lancet Oncol. 2018:19(12):1579-89.

25. Wang DY, Okoye GD, Neilan TG, Johnson DB, Moslehi JJ. Cardiovascular toxicities associated with Cancer immunotherapies. Curr Cardiol Rep. 2017; 19(3):21.

26. Denlinger CS, Ligibel JA, Are M, Baker KS, Demark-Wahnefried W, Dizon D, et al. Survivorship: immunizations and prevention of infections, version 2. 2014. J Natl Compr Cancer Netw JNCCN. 2014;12(8):1098-111.

27. Läubli H, Balmelli C, Kaufmann L, Stanczak M, Syedbasha M, Vogt D, et al. Influenza vaccination of cancer patients during PD-1 blockade induces serological protection but may raise the risk for immune-related adverse events. J Immunother Cancer. 2018;6(1):40.

28. Bersanelli M, Giannarelli D, Castrignanò P, Fornarini G, Panni S, Mazzoni F, et al. INfluenza vaccine indication during therapy with immune checkpoint inhibitors: a transversal challenge. The INVIDla study Immunotherapy. 2018; 10(14):1229-39.

29. Gopalakrishnan R, Johnson DB, York S, Neuss MN, Osterman TJ, Chism DD, et al. Impact of the influenza vaccination on cancer patients undergoing therapy with immune checkpoint inhibitors (ICI). [Internet]. Poster presented at: 2018 ASCO annual meeting; 2018 Jun 4 [cited 2018 Sep 10]; Chicago, IL,USA. Available from: http://abstracts.asco.org/214/AbstView_214_226121.html

30. Udell JA, Zawi R, Bhatt DL, Keshtkar-Jahromi M, Gaughran F, Phrommintikul A, et al. Association between influenza vaccination and cardiovascular outcomes in high-risk patients: a meta-analysis. JAMA. 2013;310(16):1711-20.

31. Mamas MA, Fraser D, Neyses L. Cardiovascular manifestations associated with influenza virus infection. Int J Cardiol. 2008;130(3):304-9.

32. Bratincsák A, El-Said HG, Bradley JS, Shayan K, Grossfeld PD, Cannavino CR. Fulminant myocarditis associated with pandemic H1N1 influenza a virus in children. J Am Coll Cardiol. 2010;55(9):928-9. 
33. Caforio ALP, Pankuweit S, Arbustini E, Basso C, Gimeno-Blanes J, Felix SB, et al. Current state of knowledge on aetiology, diagnosis, management, and therapy of myocarditis: a position statement of the European Society of Cardiology Working Group on Myocardial and Pericardial Diseases. Eur Heart J. 2013;34(33):2636-48 2648a-2648d.

34. Radin JM, Hawksworth AW, Myers CA, Ricketts MN, Hansen EA, Brice GT. Influenza vaccine effectiveness: Maintained protection throughout the duration of influenza seasons 2010-2011 through 2013-2014. Vaccine. 2016;34(33):3907-12.

35. Young B, Zhao X, Cook AR, Parry CM, Wilder-Smith A, I-Cheng MC. Do antibody responses to the influenza vaccine persist year-round in the elderly? A systematic review and meta-analysis. Vaccine. 2017;35(2):212-21.

36. Hicks KA, Tcheng JE, Bozkurt B, Chaitman BR, Cutlip DE, Farb A, et al. 2014 ACC/AHA key data elements and definitions for cardiovascular endpoint events in clinical trials: a report of the American College of Cardiology/ American Heart Association task force on clinical data standards (writing committee to develop cardiovascular endpoints data standards). J Am Coll Cardiol. 2015;66(4):403-69.

37. Neilan TG, Farhad H, Mayrhofer T, Shah RV, Dodson JA, Abbasi SA, et al. Late gadolinium enhancement among survivors of sudden cardiac arrest. JACC Cardiovasc Imaging. 2015;8(4):414-23.

38. Reynolds HR, Hochman JS. Cardiogenic shock: current concepts and improving outcomes. Circulation. 2008;117(5):686-97.

39. Epstein AE, DiMarco JP, Ellenbogen KA, Estes NAM, Freedman RA, Gettes LS, et al. 2012 ACCF/AHA/HRS focused update incorporated into the ACCF/ AHA/HRS 2008 guidelines for device-based therapy of cardiac rhythm abnormalities: a report of the American College of Cardiology Foundation/ American Heart Association task force on practice guidelines and the Heart Rhythm Society. J Am Coll Cardiol. 2013;61(3):e6-75.

40. Nichol KL, Nordin J, Mullooly J, Lask R, Fillbrandt K, Iwane M. Influenza vaccination and reduction in hospitalizations for cardiac disease and stroke among the elderly. N Engl J Med. 2003;348(14):1322-32.

41. Phrommintikul A, Kuanprasert S, Wongcharoen W, Kanjanavanit R, Chaiwarith R, Sukonthasarn A. Influenza vaccination reduces cardiovascular events in patients with acute coronary syndrome. Eur Heart J. 2011;32(14):1730-5.

42. Louria DB, Blumenfeld HL, Ellis JT, Kilbourne ED, Rogers DE. Studies on influenza in the pandemic of 1957-1958. II. Pulmonary complications of influenza. J Clin Invest. 1959;38(1 Part 2):213-65.

43. Morens DM, Taubenberger JK, Fauci AS. Predominant role of bacterial pneumonia as a cause of death in pandemic influenza: implications for pandemic influenza preparedness. J Infect Dis. 2008;198(7):962-70.

44. Vinograd I, Eliakim-Raz N, Farbman L, Baslo R, Taha A, Sakhnini A, et al. Clinical effectiveness of seasonal influenza vaccine among adult cancer patients. Cancer. 2013;119(22):4028-35.

45. Vollaard A, Schreuder I, Slok-Raijmakers L, Opstelten W, Rimmelzwaan G, Gelderblom H. Influenza vaccination in adult patients with solid tumours treated with chemotherapy. Eur J Cancer Oxf Engl 1990. 2017;76:134-43.

46. Bitterman R, Eliakim-Raz N, Vinograd I, Zalmanovici Trestioreanu A, Leibovici $\mathrm{L}$, Paul M. Influenza vaccines in immunosuppressed adults with cancer. Cochrane Database Syst Rev. 2018;2:CD008983.

47. Natarajan P, Cannon CP. Myocardial infarction vaccine? Evidence supporting the influenza vaccine for secondary prevention. Eur Heart J. 2011;32(14):1701-3.

48. Warren-Gash C, Smeeth L, Hayward AC. Influenza as a trigger for acute myocardial infarction or death from cardiovascular disease: a systematic review. Lancet Infect Dis. 2009:9(10):601-10.

49. Corrales-Medina VF, Musher DM, Shachkina S, Chirinos JA. Acute pneumonia and the cardiovascular system. Lancet Lond Engl. 2013;381(9865):496-505.

50. Guarner J, Paddock CD, Shieh W-J, Packard MM, Patel M, Montague JL, et al, Histopathologic and immunohistochemical features of fatal influenza virus infection in children during the 2003-2004 season. Clin Infect Dis Off Publ Infect Dis Soc Am. 2006:43(2):132-40.

Ready to submit your research? Choose BMC and benefit from:

- fast, convenient online submission

- thorough peer review by experienced researchers in your field

- rapid publication on acceptance

- support for research data, including large and complex data types

- gold Open Access which fosters wider collaboration and increased citations

- maximum visibility for your research: over $100 \mathrm{M}$ website views per year

At $\mathrm{BMC}$, research is always in progress.

Learn more biomedcentral.com/submissions 